\title{
The Authentic Inbred Sheath - "The Immunoglobulin"
}

\author{
Santhi Gorle ${ }^{1}$, Srikanth Adusumilli ${ }^{2}$, Rukmini Jyothirmayee Jakati ${ }^{3}$, \\ Rani Ponnaganti ${ }^{1}$, Sahithi Valluripalli ${ }^{4}$ and Sanjeev V Jakati ${ }^{5 *}$ \\ ${ }^{1}$ Senior Lecturer, KLR Lenora Institute of Dental Sciences, Rajahmundry, India \\ ${ }^{2}$ Professor, Dr Sudha and Nageshwara Rao Siddhartha Institute of Dental Sciences, \\ Vijaywada, India \\ ${ }^{3}$ Senior Lecturer, Yogita Dental College and Hospital, Khed Ratnagiri, India \\ ${ }^{4}$ Private Practioner, Rajahmundry, India \\ ${ }^{5}$ Reader, Yogita Dental College and Hospital, Khed Ratnagiri, India \\ *Corresponding Author: Sanjeev V Jakati, Reader, Yogita Dental College and \\ Hospital, Khed Ratnagiri, India.
}

DOI: $10.31080 /$ ASDS.2020.05.1015

\begin{abstract}
Humans have developed a highly specialized defense mechanism system due to continuous stimulation by foreign material from the environment. Immunoglobulins are unique structures in the body which is synthesized by plasma cells and, to some extent, by lymphocytes. They have a significant role in recognizing and protecting the body against foreign material, either present freely in the body fluids or attached to cell surfaces. Besides the plasma, immunoglobulins may be identified in other body fluids or tissues such as urine, spinal fluid, milk, saliva, tears, lymph nodes, and spleen. The plasma cells and lymphocytes produce immunoglobulins at various stages of differentiation. Immunoglobulins represent a heterogeneous population to their Physico-chemical and biological properties. Immunoglobulins are divided into five classes IgG, IgA, IgM, IgD, and IgE, which are classified based on $\mathrm{H}$ chains.
\end{abstract}

Keywords: Immunoglobulins; Pathogens; Immunity

\section{Introduction}

The continuous stimulation by the foreign materials from the environment has made vertebrates to develop a highly specialized system of defense mechanism [1]. Towards the close of the nineteenth century, the humoral basis of immunity was established by demonstrating the introduction of an antigen into an animal. Certain substances called antibodies appeared in the serum and tissue fluids and reacted with antigen specifically in some observable manner. Depending on the observable reaction produced on mixing with antigens, the antibodies were designated as agglutinins, precipitin, and so on. Sera having high antibody levels following infection or immunization, was called 'immune sera'.

Fractionation of immune sera by half-saturation with ammonium sulfate separated serum proteins into soluble albumins and in- soluble globulins. Globulins could be separated into water-soluble pseudoglobulins and insoluble euglobulins. Most antibodies were found to be euglobulins. Tiselius (1937) separated serum proteins into albumin, alpha, beta, and gamma globulins based on their electrophoretic mobilities. He showed that antibody activity was associated with the gammaglobulin fraction.

WHO in 1964 gave the generic term 'Immunoglobulin' and defined as "Immunoglobulins are proteins of animal origin endowed with known antibody activity and certain proteins related to them by chemical structure and hence antigenic specificity." Immunoglobulins provide structural and chemical concepts, while the term "antibody" is a biological and functional concept. All antibodies are immunoglobulins, but all immunoglobulins may not be antibodies [2]. They have a significant role in recognizing and protecting the body against foreign material, either present freely in the body 
fluids or attached to cell surfaces. Besides the plasma, immunoglobulins may be identified in other body fluids or tissues such as urine, spinal fluid, milk, saliva, tears, lymph nodes, and spleen. The plasma cells and lymphocytes produce immunoglobulins at various stages of differentiation.

Immunoglobulins represent a heterogeneous population for their Physico-chemical and biological properties. Immunoglobulins are divided into five classes $\operatorname{IgG}, \operatorname{IgA}, \operatorname{IgM}, \operatorname{IgD}$, and $\operatorname{IgE}$, which are classified based on H chains. (WHO 1964, Bennich., et al. 1968, Rowe and Fabey, 1965).

Immunoglobulins are multichain glycoproteins composed of two types of polypeptide chains (Edelman 1959, Edelman and Poulik 1961). Due to molecular weight differences, it was possible to isolate two types of chains heavy $(\mathrm{H})$ and light $(\mathrm{L})$. The two chains are connected covalently by disulfide bonds and form a basic unit of the immunoglobulin molecule.

Human studies have shown that periodontal patients can have circulating antibodies specific for oral bacteria and can be shown to be hypersensitive to antigens and plaque bacteria. Brandtzaeg and Kraus (1965) found that in healthy gingiva, many of the cells contained immunoglobulin G, very few contained immunoglobulin A and only rarely as immunoglobulin M. They also found that in many clinically inflamed specimens, the immunoglobulin A containing cells were over numbered, compared to the number of immunoglobulin G containing cells [3].

WHO, in 1964, defined, "Immunoglobulins are proteins of animal origin endowed with known antibody activity and certain proteins related to them by chemical structure and hence antigenic specificity".

Immunoglobulins constitute 20-25 percent of the total serum proteins. Based on physicochemical and antigenic differences, five immunoglobulins classes have been recognized-IgG, IgA, IgM, IgD, and IgE. (Both Ig and $\gamma$ are accepted abbreviations for immunoglobulins.

\section{Structure of immunoglobulin}

Edelman (1967), Nisonoff (1961), and Porter (1959) conducted studies involving the cleavage of the immunoglobulin molecule and gave a detailed picture of its structure.
The following enzymes have been examined to determine their effect upon immunoglobulin molecules: papain, pepsin, trypsin, chymotrypsin, and thermolysin. Rabbit IgG antibody to egg albumin, digested by papain in the presence of cysteine, was split into two fractions-an insoluble fraction Fc (for crystallizable) and a soluble fraction Fab (ab for antigen-binding). Each molecule of immunoglobulin is split by papain into three parts, one Fc and two Fab pieces, having a sedimentation coefficient of $3.5 \mathrm{~S}$.

Immunoglobulins are structurally glycoprotein molecules. Each molecule comprises two large, heavy chains and two small light chains. The L chain has a molecular weight of approximately 25,000 and the $H$ chain of 50,000 . The $L$ chain is joined to the $H$ chain by an S-S bond. The two H chains are joined by 1-5 S-S bonds depending on the class of immunoglobulins. Class identity is determined by class-specific sequences in the Fc region of the heavy chain, designated by Greek letters corresponding to the immunoglobulins letter designation: alpha - IgA, delta-IgD, epsilon-IgE, gamma-IgG, mu-IgM.

The L chains are present in all immunoglobulins classes. They occur as two types, kappa $(\kappa)$ and lambda $(\lambda)$. Kappa and lambda are named after Korngold and Lapari, who had originally introduced them. The kappa chains and lambda chains occur in a ratio of about 2:1 in human sera. A molecule of immunoglobulin may have either kappa or lambda chain, but both together are never present.

An antigen-binding region is formed from the amino-terminal end of both the light and heavy immunoglobulin polypeptide chains in an antibody. L chain is made up of 214 amino acid residues. 107 constitute a free carboxyl group that occurs in a constant sequence called 'constant region. In the constant region, two sequence patterns are found - those determining the kappa and lambda specificities. The portion of the amino-terminal of an antibody heavy and light chains having a variable amino acid sequence. The structure of the variable region determines the antigen specificity of the antibody. Therefore it is called the 'variable region.' Like the $\mathrm{L}$ chain $\mathrm{H}$ chain also has both 'constant' and 'Variable' regions. In the $\mathrm{L}$ chain, both constant and variable regions are of equal length. In the $\mathrm{H}$ chains, the variable region constitutes approximately only a fifth of the chain and is located at its amino-terminus. The apparent infinite range of the antibody specificity depends on the variability of the aminoacid sequences at the $H$ and L chains' variable regions, which form the antigen combining sites. 
The $\mathrm{H}$ and L chains' variable regions' amino acid sequences are not uniformly variable along their length but consist of relatively invariable and some highly variable zones. The highly variable zones numbering three in the $\mathrm{L}$ and four in the $\mathrm{H}$ chains are named hypervariable regions (or hot spots) and form the antigenbinding sites. The sites on the hypervariable regions that bind with the epitope are called 'complementarity determining regions' or CDRs. The Fc fragment is composed of the carboxyterminal portion of the $\mathrm{H}$ chains. It does not possess antigen combining activity but determines the immunoglobulin molecule's biological properties, such as complement fixation, placental transfer, skin fixation, and catabolic rate. The part of the $\mathrm{H}$ chain present in the Fab fragment is called the Fd piece. The $\mathrm{H}$ chain carries a carbohydrate moiety, which is different for each class of immunoglobulins.

Each immunoglobulin peptide chain has internal S-S links and interchain S-S bonds, which interlink the $\mathrm{H}$ and $\mathrm{L}$ chains. These interchain S-S bonds form loops in the peptide chain, and each of the loops is folded compactly to form a globular domain, each domain having a separate function. The variable region domains VL and $\mathrm{VH}$ are responsible for the formation of a specific $\mathrm{F}_{\mathrm{ab}}$ site. The $\mathrm{CH}^{2}$ region in IgG binds Clq in the classical complement sequence, and the $\mathrm{CH}^{3}$ domain mediates adherence to the surface of the monocyte. The $\mathrm{H}$ chain in the $\mathrm{C}$ region areas between the first and second $\mathrm{C}$ region domains $\left(\mathrm{CH}^{1}\right.$ and $\left.\mathrm{CH}^{2}\right)$ is called the hinge region and is more flexible and more exposed to enzymes and chemicals.

Immunoglobulin classes

Five immunoglobulins classes have been recognized and were named IgG, IgA, IgM, IgD, and IgE in the descending order of concentration.

- IgG: This is a major serum immunoglobulin, constituting about $80 \%$ of the total immunoglobulins, with a molecular weight of 150,000 (7S). The normal serum concentration of IgG is about 8-16 mg per ml. IgG may occasionally exist in a polymerised form. It has a half-life of approximately 23 days. It is distributed approximately equally between the intravascular and extravascular compartments. The catabolism of IgG is unique in which it varies with serum concentration, and it is the only maternal immunoglobulin that is normally transported across the placenta and provides natural passive immunity to the newborn and is not synthesised by the fetus in any sig- nificant amount. IgG binds to microbes and enhances their phagocytosis. IgG participates in most immunological reactions such as complement fixation, precipitation, and neutralisation of toxins and viruses. Furthermore, it may be considered a general-purpose antibody, protective against infectious agents active in the blood and tissues. Four subclasses of IgG have been recognised (IgG 1, IgG2, IgG3, IgG4), each possessing a distinct type of gamma chain, identifiable with specific antisera. The four IgG subclasses are distributed in human serum in the approximate proportions of 65 percent, 23 percent, 8 percent, and 4 percent, respectively.

- IgA: IgA is the second major serum immunoglobulin class constituting about $10-13 \%$ of serum. The normal serum level is $0.6-4.2 \mathrm{mg} / \mathrm{ml}$, which has a half-life of 6-8 days. It is a major immunoglobulin in the colostrum, saliva, and tears. IgA occurs in two forms: Serum IgA and secretory IgA. Serum IgA is principally a monomeric $7 \mathrm{~S}$ molecule (MW 160,000) found on mucosal surfaces and secretions. A dimer formed by two monomer units joined at theircarboxy terminals by a glycopeptide termed the J chain (J for joining )This is called the secretory IgA (SIgA). Dimeric SIgA is synthesised by plasma cells situated near the mucosal or glandular epithelium. SIgA contains another glycine-rich polypeptide called the secretory component or secretory piece. The secretory piece is believed to protect IgA from denaturation by bacterial proteases. In sites such as the intestinal mucosa, which have a rich and varied bacterial flora. SIgA is a much larger molecular weight of SIgA is about 400,000. IgA antibodies inhibit microorganisms' adherence to mucosal cells' surface by covering the microorganisms and preventing their entry into tissues. IgA cannot fix the complement but can activate the alternative complement pathway, promoting phagocytosis and causing intracellular death of microorganisms. Two subclasses of IgA are IgA1 and IgA2. IgA2 lacks interchain S-S bonds between the $\mathrm{H}$ and $\mathrm{L}$ chains. Though IgA2 is a minor serum component of IgA, it is abundant in the secretions.

- IgM: IgM constitutes 5-8 \% of serum immunoglobulins with a normal range of $0.5-2 \mathrm{mg} / \mathrm{ml}$, which has a half-life of about five days. It is a heavy molecule (19S; moL wt 
900,000 to $1,000,000$, hence called 'the millionaire molecule'). IgM molecules are polymers of five four-peptide subunits, each bearing an extra $\mathrm{CH}$ domain. The polymerisation of the subunits depends upon the presence of the J chain. Most of the serum IgM (80\%) is intravascular in distribution and is also the earliest immunoglobulin to be synthesised by the fetus by about 20 weeks of age. Serum IgM is not transported across the placenta. The presence of IgM in the fetus or newborn indicates an intrauterine infection, and its detection is useful in the diagnosis of congenital infections such as syphilis, rubella, HIV infection, and toxoplasmosis. IgM antibodies are relatively short-lived, and their detection in serum indicates recent infection. The iso-hemagglutinins (anti-A, anti-B) and many other natural antibodies to microorganisms are usually IgM. The unique features of IgM are to protect microorganisms and other large antigens that have repeating antigenic determinants on their surface. Singlemolecule of IgM can cause immune heamolysis, whereas 1000 IgG molecules are needed for the same effect. Itis 500-1000 times more effective than IgG in opsonisation, 100 times more effective in bactericidal action, and about 20 times in bacterial agglutination. However, in the neutralisation of toxins and viruses, it is less active than IgG. IgM is believed to be responsible for protection against blood-borne infections. IgM deficiency is often associated with septicemias and is the major antibody receptor on $\mathrm{B}$ lymphocytes' surface for antigen recognition.

- IgD: IgD is structurally similar to IgG and is present in a concentration of about $3 \mathrm{mg} / 100 \mathrm{ml}$ of serum, and it is mostly intravascular. It has a half-life of about three days with a molecular weight of about 180,000-200,000D. IgD occurs on the surface of unstimulated B lymphocytes, and it serves as a recognition receptor for antigens. A combination of the cell membrane-bound IgD or IgM with the corresponding antigen leads to specific stimulation of the B cell activation and cloning to produce antibody or suppression.

- IgE: This immunoglobulin was discovered in 1966 by Ishizaka. It is an 8S molecule (MW about 190,000)with a half-life of about two days, which resembles IgG structurally. It exhibits unique properties such as heat lability (in- activated at $56^{\circ} \mathrm{C}$ in one hour) and affinity for the surface of tissue cells (particularly mast cells) of the same species (homocytotropism), which mediates the Prausnitz Kustner reaction. It does not pass the placental barrier and is mostly extravascular in distribution. The normal serum contains only traces, but elevated levels are seen in atopic conditions such as asthma, hay fever, and eczema. $\operatorname{IgE}$ is chiefly produced in the mucosa of respiratory and intestinal tracts and is also responsible for the Type-1hypersensitivity. The physiological role of IgE is to protect against pathogens by mast cell degranulation and release of inflammatory mediators. It is also believed to have a special role in defense against helminthic infections.

However, IgG defense the body fluids, whereas IgA defends the body surfaces. IgM protects the bloodstream while IgE mediates hypersensitivity. $\operatorname{IgD}$ is a recognition molecule on the surface of $\mathrm{B}$ lymphocytes [2].

The immune system has evolved to protect us from pathogens. A highly discriminatory immune system is basic for survival. The immune system's primary function is to eliminate infectious agents and minimize the damage caused by them. Some pathogens evoke 'humoral immunity'; immunoglobulins are an essential component of humoral immunity. They are present in serum, tissue fluid, or on cell membranes. Among the five classes of Immunoglobulin, the immunoglobulin IgG is the predominant immunoglobulin in normal human serum, and IgA is predominant in seromucous secretions. Several methods to measure these antibodies of which immunoassays, immunoelectrophoresis, radioimmunoassay, ELISA are common. Antibody clearly plays a crucial role in dealing with the pathogens and this important function is performed mainly by IgG and IgA. IgA deficiency predisposes to oral mucosal infections [4]. Because IgG is the immunoglobulin class found in the highest blood concentration, it plays a major role in antibody-mediated defense mechanisms.

\section{Role of immunoglobulins in periodontitis}

Periodontal disease is one of the most prevalent diseases worldwide, which cause a serious consequence with loss of the periodontal supporting structures, which includes gingiva, cementum, periodontal ligament, and alveolar bone. The infection level can range from gingivitis, which is the inflammation of the gingiva, all the way to periodontitis results in loss of the tooth. Although the 
subgingival microbes initiate periodontitis, it is generally accepted that mediators of connective tissue breakdown are generated to a large extent by the host response to a pathogenic infection; in a susceptible host, microbial virulence factors trigger the release of host-derived enzymes and pro-inflammatory cytokines that can lead to colonial tissue destruction. The overproduction of pro-inflammatory mediators $\mathrm{PGE}_{2}$ and $\mathrm{CD} 40$ in periodontitis is induced due to antibody $\beta_{1}$-AR interaction [5].

The implication of periodontal microbes associated with endotoxin products on the induction of the innate immune response, cells like receptors signaling generation of pathogen-associated molecular patterns (PAM's) and their role in periodontal disease pathogenesis is crucial to the extent of disease severity. The majority of plasma cells and IgG-bound lymphocytes in periodontitis have cell-associated immunoglobulins IgG1, IgG3, or IgG4 subclasses. Immunoglobulins which form immune complexes are known to provoke complement fixation or stimulate the release of other mediators. The predominance of IgG in gingival organ culture supernatants and the statistically significant findings showed that the overall mean levels of IgG between mild gingivitis and periodontitis and between severe periodontitis suggested a possible indicator of periodontal disease. The chronic periodontitis, generalized aggressive periodontitis patients, has an imbalance in the host response, with reduced IL-10 and IgG levels and increased periodontal pathogens ${ }^{6}$. There are elevated levels of serum IgG to P.g and A.a are associated with coronary artery atherosclerosis, which reflects a direct role for periodontal infection or a role for the host response to infection in coronary atherosclerosis, particularly in patients with type 1 diabetes [7].

There is a correlation of IgG response to bacterial infection level in Generalized aggressive periodontitis [8]. The Chronic Periodontitis patients produced higher levels of total serum IgG and IgG specific for P. Gingivalis [9]. The predominant organisms in the pathogenesis of periodontal disease being Porphyromonas gingivalis (P. g), Aggregatibacter actinomycetemcomitans (A. a) increase in the number of these periodontal pathogens showed an increase in salivary Ig A and Ig G $[10,11]$. Serum levels of IgG antibodies against A. a or P. $g$ in periodontitis stable patients were higher than those in patients with active periodontitis. This indicates that elevated levels of IgG antibody against A. a and P.g have a detectable protective effect against periodontal infections with these microorganisms $[12,13]$.
IgA and IgG antibody levels were higher in the pathogen carriers than the non-carriers and clearly higher in the carriers with periodontal pockets than the carriers without periodontal pockets. The antibody levels and the numbers of corresponding bacteria in saliva had a positive association, which was not associated with the disease [14]. Lindsay M. Hall., et al. studied the antibody response of Tannerella forsythia bacterial surface protein A (Tf BspA) in periodontal disease. They suggested that anti-BspA IgG might have a protective function in periodontal disease by minimizing the loss of tooth attachment tissue [15].

A study conducted by Graswinckel JEM., et al. showed that nonsmoker periodontitis patients have higher levels of total IgG1 and IgG2 levels than smoker periodontitis patients [16]. Smoking is the environmental factor that influences the disease progression of periodontal disease. Immunoglobulin production and B cell function decreased in smoking patients than non smokers $[17,18]$. Evidence suggests that anti-HA2 IgG antibodies correspond directly with periodontal health, possibly through their ability to neutralize $P$. gingivalis hemoglobin capture [19]. The major factors regulating the destruction of tooth-supporting periodontal structures are inflammatory mediators released by host cells. Gaffen and Hajishengallis describe periodontal health as "a dynamic state where the activity of pro-inflammatory/antimicrobial cytokines to control infection is optimally balanced by anti-inflammatory mechanisms to prevent unwarranted inflammation". The immune response itself may contribute significantly to the periodontal destruction, sometimes even more than the pathogens. IgG's predominance in gingival organ culture supernatants and the statistically significant findings that the overall mean levels of IgG between mild gingivitis and periodontitis and between severe periodontitis and periodontitis suggested a possible indicator of periodontal disease [20].

\section{Conclusion}

In conclusion, immunoglobulin is an intrinsic molecule with a protective function in the body by recognizing the antigens and immunizing the body for the antigen's imminent charge. It increases disease conditions, and some immunoglobulins are raised for specific diseases and act as a marker in diagnosing the disease. Hence, the knowledge of immunoglobulin to the various disease association helps in the molecular basis of the many diseases' perception. 


\section{Bibliography}

1. J Mestecky. "Structure of antibodies". Journal of Oral Pathology 1 (1972): 288-300.

2. R Ananthanarayana and CKJ Paniker. Textbook of microbiology 7th ed: 85-91.

3. Robert J Genco. "Immunoglobulins and periodontal disease". Journal of Periodontology 41 (1970): 196-201.

4. Jorgensen GH., et al. "Immunoglobulin A deficiency and oral health status: a case-control study". Journal of Clinical Periodontology 37 (2010): 1-8.

5. Sterin-Borda L., et al. "Autoantibodies to $\beta 1$-adrenoceptors in human chronic periodontitis induce overexpression of fibroblast CD40 and trigger prostaglandin E2 generation". Journal of Periodontal Research 44 (2009): 330-337.

6. Casarin RCV., et al. "Levels of Aggregatibacter actinomycetemcomitans, Porphyromonas gingivalis, inflammatory cytokines and species-specific immunoglobulin $\mathrm{G}$ in generalized aggressive and chronic periodontitis". Journal of Periodontal Research 45 (2010): 635-642.

7. Colhoun HM., et al. "Antibodies to periodontal pathogens and coronary artery calcification in type 1 diabetic and nondiabetic subjects". Journal of Periodontal Research 43 (2008): 103110.

8. Saraiva L., et al. "IgG sera levels against a subset of periodontopathogens and severity of disease in aggressive periodontitis patients: a crosssectional study of selected pocket sites". Journal of Clinical Periodontology 41 (2014): 943-951.

9. Trindade SC., et al. "Induction of interleukin (IL)-1b, IL-10, IL-8 and immunoglobulin $\mathrm{G}$ by Porphyromonas gingivalis $\mathrm{HmuY}$ in humans". Journal of Periodontal Research 47 (2012): 27-32.

10. Pushpa S., et al. "Correlation of salivary immunoglobulin A against lipopolysaccharide of Porphyromonas gingivalis with clinical periodontal parameters". Contemporary Clinical Dentistry 6 (2015): 305-308.

11. John M Liljestrand., et al. "Combining Salivary Pathogen and Serum Antibody Levels Improves Their Diagnostic Ability in Detection of Periodontitis". Journal of Periodontology 85 (2014): 123-131.

12. Rams TE., et al. "Actinobacillus actinomycetemcomitans and Porphyromonas gingivalis subgingival presence, species-specific serum immunoglobulin $\mathrm{G}$ antibody levels, and periodon- titis disease recurrence". Journal of Periodontal Research 41 (2006): 228-234.

13. Sakai Y., et al. "Porphyromonas gingivalis- specific IgG subclass antibody levels as immunological risk indicators of periodontal bone loss". Journal of Clinical Periodontology 28 (2001): 853-859.

14. Pussinen PJ., et al. "Periodontal pathogen carriage, rather than periodontitis, determines the serum antibody levels". Journal of Clinical Periodontology 38 (2011): 405-411

15. Lindsay M Hall., et al. "Levels of Serum Immunoglobulin G Specific to Bacterial Surface Protein A of Tannerella forsythia are Related to Periodontal Status". Journal of Periodontology 2012 83 (2012): 228-234.

16. Graswinckel JEM., et al. "Plasma antibody levels in periodontitis patients and controls". Journal of Clinical Periodontology 31 (2004): 562-568.

17. Apatzidou DA., et al. "Impact of smoking on the clinical, microbiological, and immunological parameters of adult patients with periodontitis". Journal of Clinical Periodontology 32 (2005): 973-983.

18. Hamdan S Al-Ghamdi and Sukumaran Anil. "Serum antibody levels in smoker and non smoker saudi subjects with chronic periodontitis". Journal of Periodontology 78 (2007): 10431050.

19. Decarlo AA., et al. "Serum antibodies against the hemoglobinbinding domain (HA2) of Porphyromonas gingivalis". Journal of Periodontal Research 39 (2004): 228-235.

20. Reena Kulshrestha., et al. "Role of immunoglobulin in periodontitis". Journal of Pure and Applied Microbiology 7.1 (2013): 673-676.

\section{Assets from publication with us}

- Prompt Acknowledgement after receiving the article

- Thorough Double blinded peer review

- Rapid Publication

- Issue of Publication Certificate

- High visibility of your Published work

Website: www.actascientific.com/

Submit Article: www.actascientific.com/submission.php

Email us: editor@actascientific.com

Contact us: +919182824667 